\title{
Impact of Admission Blood Glucose Added on GRACE Risk Score for All-Cause In-Hospital Mortality in Patients with Acute Coronary Syndrome
}

\author{
Md. Mesbahul Islam ${ }^{1}$, Mohsin Ahmed ${ }^{2}$, Abdul Wadud Chowdhury $^{3}$, Mohammad Ali $^{4}$, Khandakar Abu Rubayat $^{5}$
}

\begin{abstract}
:
Background: Abnormal glucose metabolism is a predictor of worse outcome after acute coronary syndrome (ACS). However, this parameter is not included in risk prediction scores, including GRACE risk score. We sought to evaluate whether the inclusion of blood glucose at admission in a model with GRACE risk score improves risk stratification. Objectives: To assess whether inclusion of admission blood glucose in a model with GRACE risk score improves risk stratification of ACS patients admitted in a tertiary hospital of Bangladesh. Methods: This cross sectional comparative study was carried out in the department of cardiology, Dhaka Medical College Hospital (DMCH), Dhaka between May 2016 to April 2017. Data were collected from ACS patients admitted at CCU, DMCH who fulfilled inclusion and exclusion criteria. GRACE score was calculated for each patient. The predictive value of death by GRACE score was compared with the predictive value of combined GRACE score + admission blood sugar. Comparison between these results in two groups were done by unpaired t-test, analysis was conducted SPSS-22.0 for windows software. The significance of the results was determined in $95.0 \%$ confidence interval and a value of p $<0.05$ was considered to be statistically significant. Results: A total of 249 cases of ACS patients were selected. Most of the patients belonged to $5^{\text {th }}$ and $6^{\text {th }}$ decades $25.3 \%$ vs $37.3 \%$ and the mean age was $55.7 \pm 11.7$ years. Most of the patients were male. High GRACE risk score $(\geq 155)$ and elevated admission blood sugar $(\geq 11)$ was found significantly higher in-hospital death whereas
\end{abstract}

only high GRACE risk score $(\geq 155)$ and normal admission blood sugar $(<11)$ was found non significant regarding in-hospital death. Test of validity showed sensitivity of GRACE risk score regarding in-hospital death was $85.29 \%$, specificity $57.7 \%$, accuracy $61.4 \%$, positive and negative predictive values were $24.2 \%$ and $96.1 \%$ respectively. The sensitivity of GRACE risk score + admission blood sugar regarding in-hospital death was $85.29 \%$, specificity $62.33 \%$, accuracy $65.46 \%$, positive and negative predictive values were $26.36 \%$ and $96.4 \%$ respectively. Receiver-operator characteristic (ROC) were constructed using GRACE score and GRACE score + admission blood sugar of the patients with in-hospital death, which showed the sensitivity and specificity of GRACE score for predicting in-hospital death were found to be $79.4 \%$ and $58.1 \%$, respectively. Whereas after adding admission blood sugar value to GRACE score both the sensitivity and specificity increased to $82.4 \%$ and $58.6 \%$ respectively in this new model. Logistic regression analysis of in-hospital mortality with independent risk factors showed GRACE score $(\geq 155)+$ admission blood sugar ( $\geq 11.0 \mathrm{mmol} / \mathrm{l})$ was more significantly associated with in-hospital mortality ( $P$ $=0.001, \mathrm{OR}=6.675,95 \% \mathrm{Cl} 2.366-13.610)$. Conclusion: In patients with the whole spectrum of acute coronary syndrome admission blood glucose can add prognostic information to the established risk factors with the GRACE risk score.

Keywords: Admission blood glucose, GRACE risk score, acute coronary syndrome.

(Bangladesh Heart Journal 2018; 33(2): 94-99)

1. Registrar, Department of Cardiology, Ibn Sina Specialized Hospital, Dhanmondi, Dhaka, Bangladesh.

2. Associate Professor, Dept. of Cardiology, National Institute of Cardiovascular Diseases, Dhaka, Bangladesh.

3. Professor, Dept. of Cardiology, Dhaka Medical College, Dhaka, Bangladesh.

4. Specialist, Department of Cardiology, Bangladesh specialized Hospital, Shyamoli, Dhaka, Bangladesh.

5. MD, final Part, Dhaka Medical College, Dhaka, Bangladesh.

Address of Correspondence: Dr. Md. Mesbahul Islam, Registrar, Department of Cardiology, Ibn Sina Specialized Hospital, Dhanmondi, Dhaka, Bangladesh. Cell no. +8801710834077, Email: dmesbah37@gmail.com

DOI: http://dx.doi.org/10.3329/bhj.v33i2.39304

Copyright $\odot 2017$ Bangladesh Cardiac Society. Published by Bangladesh Cardiac Society. This is an Open Access articles published under the Creative Commons Attribution-NonCommercial 4.0 International License (CC BY-NC). This license permits use, distribution and reproduction in any medium, provided the original work is properly cited and is not used for commercial purposes. 


\section{Introduction:}

Coronary artery disease (CAD) is an increasingly important medical and public health problem and is the leading cause of mortality in Bangladesh as well. Like other South Asians, Bangladeshis are unduly prone to develop CAD, which is often premature in onset, follows a rapidly progressive course and angiographically more severe. ${ }^{1}$ Some of the independent predictors of early death from STEMI include age, Killip class, time to reperfusion, cardiac arrest, tachycardia, hypotension, anterior infarct location, prior infarction, diabetes mellitus, smoking status, renal function and biomarker findings. ${ }^{2}$ The presence of diabetes doubled the age adjusted risk for cardiovascular disease in men and tripled it in women in the Framingham Heart Study and it remained an independent risk factor even after adjusting for age, hypertension, smoking, hyperlipidemia, and left ventricular hypertrophy. ${ }^{3}$ Acute hyperglycemia is common in patients with STEMI even in the absence of a history of type $2 \mathrm{DM}$. Hyperglycemia is encountered in up to $50 \%$ of all STEMI patients, whereas previously diagnosed DM is present in only $20 \%$ to $25 \%$ of STEMI patients. ${ }^{4}$ In the clinical practice, admission plasma glucose is used as a measure of Acute Hyperglycemia and Hemoglobin Alc (HbA1c) for Chronic Hyperglycemia. Acute Hyperglycemia was defined as plasma glucose $>198 \mathrm{mg} / \mathrm{dl}$ $(11 \mathrm{mmol} / \mathrm{L})$ at admission, regardless of diabetic status. ${ }^{5}$

Elevated plasma glucose and glycated hemoglobin levels on admission are independent prognostic factors of both in-hospital and long term outcome regardless of diabetic status. ${ }^{6}$ For every $18 \mathrm{mg} / \mathrm{dL}$ increase in glucose level, there is a $4 \%$ increase in mortality in non-diabetic subjects. ${ }^{7}$ Admission glucose has been identified as a major independent predictor of both in-hospital Congestive Heart Failure and Mortality in STEMI. ${ }^{8}$ Hyperglycemia at presentation, while often reflecting undiagnosed and persisting abnormalities of glucose handling, may also represent a transient stress response mediated through the autonomic nervous system with release of catecholamines and adrenal corticosteroids. ${ }^{9}$ This catecholamines response occurs early, is restricted to the first five days and is proportional to the size of infarction, being associated with faster heart rate, poorer Killip class and lower ejection fraction on discharge. ${ }^{10}$ Hyperglycemia is associated with large infarction and depressed left ventricular function, heart failure on admission and elevated Brain Natriuretic Peptide. ${ }^{11}$ On the other hand whatever the cause of hyperglycemia in acute myocardial infarction, it has got a detrimental effect on myocardium itself. Effects of hyperglycemia include the promotion of oxidative stress, impairment of endothelial function, promotion of coagulation, non-enzymatic glycation of platelet glycoproteins with abrupt changes in agreeability, amplification of inflammation, suppression of immunity and direct toxicity to myocytes and promotion of apoptosis. Acute hyperglycemia has been shown to impair ischemic preconditioning, attenuate the protective effect of preinfarction angina on microvascular function and reduce the effectiveness of collateral blood supply into ischemic zones. ${ }^{12}$

An ACS may take the form of an ST-elevation myocardial infarction (STEMI), a non-ST-elevation myocardial infarction (NSTEMI), or unstable angina. The Global Registry of Acute Coronary Events (GRACE) risk score is a validated and established score for risk stratification of patients with acute coronary syndromes, obtained from a multicentre registry. ${ }^{13}$ Though elevated plasma glucose is an independent prognostic factor is not included in this risk scoring system. We sought to evaluate whether inclusion of admission blood glucose in a model with GRACE risk score improves risk stratification.

\section{Methodology:}

The cross-sectional comparative study was carried out in the Department of Cardiology, Dhaka Medical College Hospital, Dhaka from May 2016 to April 2017. All the patients of Acute Coronary Syndrome (STEMI, NSEMI, UA) after exclusion and inclusion criteria were taken as sampling population. Patients/attendance was briefed about the study and consent was taken. Brief history was taken included with symptoms and risk factors. Relevant physical examination, 12 lead ECG was done on admission and routinely thereafter. Blood glucose level e"11.0 mmol/l or $198 \mathrm{mg} / \mathrm{dl}$ was considered as admission hyperglycemia. Baseline investigations including-cardiac biomarkers, serum creatinine, lipid profile and echocardiography were done for each patient. All the above informations were recorded in a data collection form consisting of relevant questionnaire. GRACE score for each patient was calculated by using the online GRACE risk calculator by eight variables taken into account: patient age, heart rate, systolic blood pressure, serum creatinine, Killip heart failure class, the existence or not of cardiac arrest at admission, any deviations of the ST segment and cardiac enzyme levels. Then occurrence of in-hospital death and complications (acute LVF, cardiogenic shock, ventricular tachycardia, ventricular fibrillation, asystole and AV block) were recorded. The predictive value of death by GRACE score was compared with the predictive value of combined GRACE score + admission blood sugar. Comparison between these results in two groups were done by chi square test, analysis was conducted SPSS-23.0 for Windows software. The significance of the results was determined in $95.0 \%$ confidence interval and a value of $p$ $<0.05$ was considered to be statistically significant. 


\section{Results:}

Most of the patients belonged to $5^{\text {th }}$ and $6^{\text {th }}$ decades $25.3 \%$ vs $37.3 \%$ and the mean age was $55.7 \pm 11.7$ years. Most of the patients were male. Male female ratio was 2.8:1. (Table I). High GRACE risk score $(\geq 155)$ and elevated admission blood sugar ( $\geq 11$ ) was found significantly higher in-hospital death whereas only high GRACE risk score $(\geq 155)$ and normal admission blood sugar $(<11)$ was found non significant regarding in-hospital death (Table II). Test of validity showed the sensitivity of GRACE risk score regarding inhospital death was $85.29 \%$, specificity $57.7 \%$, accuracy $61.4 \%$, positive and negative predictive values were $24.2 \%$ and $96.1 \%$ respectively. The sensitivity of GRACE risk score plus admission blood sugar regarding in-hospital death was $85.29 \%$, specificity $62.33 \%$, accuracy $65.46 \%$, positive and negative predictive values were $26.36 \%$ and $96.4 \%$ respectively (Table III). Receiver-operator characteristic (ROC) curves were constructed using GRACE score and GRACE score + admission blood sugar of the patients with in-hospital death, which showed the sensitivity and specificity of GRACE score for predicting in-hospital death were found to be $79.4 \%$ and $58.1 \%$, respectively. Whereas after adding admission blood sugar value to GRACE score the sensitivity increased to $82.4 \%$ and specificity increased to $58.6 \%$ at the same cut off value (Table IV). Logistic regression analysis of in-hospital mortality with independent risk factors showed
GRACE score $(\geq 155)+$ admission blood sugar $(\geq 11.0 \mathrm{mmol} /$ I) was more significantly associated with in-hospital mortality (OR = 6.675, 95\% Cl 2.366-13.610). (Table V).

Table-I

Demographic characteristics of the study patients $(n=249)$

\begin{tabular}{lcc}
\hline Age (years) & $\begin{array}{c}\text { Number of } \\
\text { patients }\end{array}$ & Percentage \\
\hline$\geq 30$ & 6 & 2.4 \\
$31-40$ & 24 & 9.6 \\
$41-50$ & 63 & 25.3 \\
$51-60$ & 93 & 37.3 \\
$61-70$ & 42 & 16.9 \\
$>70$ & 21 & 8.4 \\
Mean \pm SD & & \\
Sex & & \\
$\quad$ Male & 184 & 73.9 \\
$\quad$ Female & 65 & 26.1 \\
Smoking & 134 & 53.8 \\
Diabetes mellitus & 43 & 17.3 \\
Hypertension & 131 & 52.6 \\
Dyslipidemia & 34 & 13.7 \\
Family H/o CAD & 64 & 25.7 \\
\hline
\end{tabular}

Table-II

Association of in-hospital death with GRACE risk score plus admission blood sugar $(n=249)$

\begin{tabular}{|c|c|c|c|c|c|}
\hline \multirow{3}{*}{$\begin{array}{l}\text { GRACE risk score plus } \\
\text { admission blood sugar }\end{array}$} & \multicolumn{4}{|c|}{ In-hospital death } & \multirow[t]{3}{*}{$p$ value } \\
\hline & \multicolumn{2}{|c|}{ Yes(n=34) } & \multicolumn{2}{|c|}{$\mathrm{No}(\mathrm{n}=215)$} & \\
\hline & $\mathrm{n}$ & $\%$ & $\mathrm{n}$ & $\%$ & \\
\hline $\begin{array}{l}\text { GRACE risk score } \geq 155 \text { and admission } \\
\text { blood sugar } \geq 11 \mathrm{mmol} / \mathrm{L}\end{array}$ & 29 & 85.3 & 81 & 37.7 & $0.001^{\mathrm{s}}$ \\
\hline $\begin{array}{l}\text { GRACE risk score } \geq 155 \text { and admission } \\
\text { blood sugar }<11 \mathrm{mmol} / \mathrm{L}\end{array}$ & 11 & 32.4 & 50 & 23.3 & $0.252^{\mathrm{ns}}$ \\
\hline
\end{tabular}

$\mathrm{s}=$ significant, $\mathrm{ns}=$ not significant

$P$ value reached from Chi square test

Table III

Sensitivity, specificity of predicted in-hospital death between GRACE risk score and GRACE risk score plus admission blood sugar.

\begin{tabular}{|c|c|c|c|c|c|}
\hline & Sensitivity & Specificity & Accuracy & PPV & NPV \\
\hline GRACE risk score $(\geq 155)$ & 85.29 & 57.7 & 61.4 & 24.2 & 96.1 \\
\hline $\begin{array}{l}\text { GRACE risk score }(\geq 155) \text { and } \\
\text { admission blood sugar } \\
(\geq 11 \mathrm{mmol} / \mathrm{L})\end{array}$ & 85.29 & 62.33 & 65.46 & 26.36 & 96.4 \\
\hline
\end{tabular}




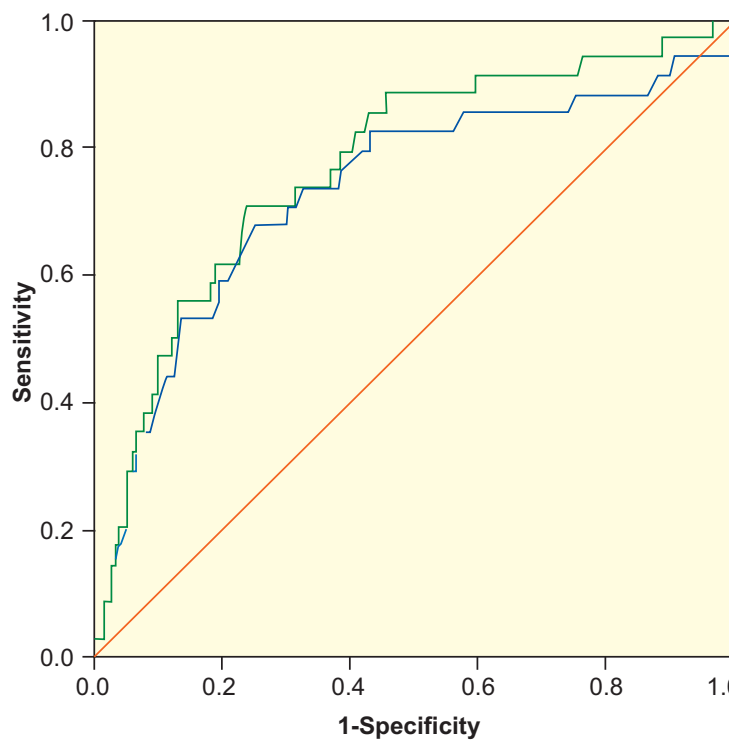

Source of the Curve

- Gracescore

_ Grace_score_and_RBS

- Reference Line

Fig.-1: Receiver-operator characteristic curves of GRACE score and GRACE score + admission blood sugar.

Table-IV

Receiver-operator characteristic (ROC) curve of GRACE score and GRACE score + admission blood sugar for prediction of in-hospital death

\begin{tabular}{|c|c|c|c|c|c|c|}
\hline & \multirow{2}{*}{$\begin{array}{l}\text { Cut off } \\
\text { value }\end{array}$} & \multirow[t]{2}{*}{ Sensitivity } & \multirow[t]{2}{*}{ Specificity } & \multirow{2}{*}{$\begin{array}{l}\text { Area under the } \\
\text { ROC curve }\end{array}$} & \multicolumn{2}{|c|}{$95 \%$ Confidence interval $(\mathrm{Cl})$} \\
\hline & & & & & Lower bound & Upper bound \\
\hline GRACE score & $\geq 155$ & 79.4 & 58.1 & 0.731 & 0.627 & 0.836 \\
\hline $\begin{array}{l}\text { GRACE score + admission } \\
\text { blood sugar }\end{array}$ & $\geq 155$ & 82.4 & 58.6 & 0.769 & 0.680 & 0.859 \\
\hline
\end{tabular}

Table-V

Multi variable logistic regression analysis $(n=249)$

\begin{tabular}{lcccc}
\hline & Adjusted & \multicolumn{2}{c}{$95 \% \mathrm{Cl}$} & $\mathrm{P}$ \\
\cline { 2 - 4 } & OR & Lower & Upper & Value \\
\hline Age $(>50$ years) & 0.333 & 0.114 & 0.975 & $0.045^{\mathrm{s}}$ \\
GRACE score $(\geq 155)$ & 5.840 & 1.407 & 24.235 & $0.015^{\mathrm{s}}$ \\
Admission blood sugar ( $\geq 11.0$ mmol/I) & 1.794 & 0.633 & 5.083 & $0.271^{\mathrm{ns}}$ \\
GRACE score $(\geq 155)+$ admission blood sugar $(\geq 11.0 \mathrm{mmol} / \mathrm{l})$ & 6.675 & 2.366 & 13.610 & $0.001^{\mathrm{s}}$ \\
Diabetes mellitus & 0.171 & 0.020 & 1.471 & $0.108^{\mathrm{ns}}$ \\
Smoking & 1.768 & 0.676 & 4.624 & $0.246^{\mathrm{ns}}$ \\
Dyslipidaemia & 0.417 & 0.077 & 2.253 & $0.310^{\mathrm{ns}}$ \\
Heart failure (Killip class II-IV) & 1.842 & 0.528 & 6.434 & $0.338^{\mathrm{ns}}$ \\
Ejection fraction $(<30 \%)$ & 0.133 & 0.001 & 1.210 & $0.998^{\mathrm{ns}}$ \\
\hline
\end{tabular}

$\mathrm{s}=$ significant, $\mathrm{ns}=$ not significant

Multivariable logistic regression analysis was performed 


\section{Discussion:}

This cross sectional comparative observational study was conducted in the Department of Cardiology, Dhaka Medical College Hospital, Dhaka, over a period of one year from May 2016 to April 2017. The main objective was to assess whether inclusion of admission blood glucose in a model with GRACE risk score improves risk stratification of ACS patients. For this purpose 249 patients with acute coronary syndrome were included according to exclusion and inclusion criteria. In our study most of the patients belongs to $5^{\text {th }}$ and $6^{\text {th }}$ decades $25.3 \%$ vs $37.3 \%$ and the mean age was $55.7 \pm 11.7$ years. Nearly similar results were observed by Mudespacher et al. ${ }^{14}$ and Timoteo et al. ${ }^{15}$. Most of the patients were male. Male female ratio was 2.8:1. High GRACE risk score (e"155) and elevated admission blood sugar (e"11) was found significantly higher in-hospital death whereas only high GRACE risk score (e"155) and normal admission blood sugar $(<11)$ was found non significant regarding in-hospital death.

Several possible mechanisms may explain this observation. First, hyperglycemia is a reflection of relative insulin deficiency, which is associated with increased lipolysis and excess circulating free fatty acids; this effect may be exaggerated in cases of acute stress such as myocardial infarction. ${ }^{16,17}$ Free fatty acids, although normally the substrate of choice for healthy myocardium, are toxic to ischemic myocardium and may lead to damaged cardiaccell membranes, calcium overload, and arrythmias. ${ }^{18}$ Insulin deficiency may also limit the ability of cardiac muscle to take up glucose for anaerobic metabolism. Second, acute hyperglycemia may precipitate an osmotic diuresis. The resulting volume depletion may interfere with the FrankStarling mechanism, an important compensatory mechanism for the failing left ventricle in which increased end-diastolic volume leads to increased stroke volume. ${ }^{19,20}$ Third, stress hyperglycemia may be a marker of more extensive cardiac damage in acute myocardial infarction. ${ }^{21}$ More extensive cardiac damage may lead to a greater rise in stress hormones (promoting glycogenolysis and hyperglycemia) and may also increase the risk of congestive heart failure and mortality. Thus, stress hyperglycemia could simply be an epiphenomenon reflecting the most severe cardiac damage. Fourth, patients who develop stress hyperglycemia are likely to be dysglycemic when not stressed. Patients with dysglycemia are at a higher risk of cardiovascular disease than patients who have normal blood glucose ${ }^{22}$ and may have a worse prognosis after acute myocardial infarction because of more extensive underlying coronary artery disease.

In this study we found that the sensitivity of GRACE risk score regarding in-hospital death was $85.29 \%$, specificity was $57.7 \%$, accuracy was $61.4 \%$, positive and negative predictive values were $24.2 \%$ and $96.1 \%$ respectively. The sensitivity of GRACE risk score + admission blood sugar regarding in-hospital death was $85.29 \%$, specificity was $62.33 \%$, accuracy was $65.46 \%$, positive and negative predictive values were $26.36 \%$ and $96.4 \%$ respectively. Similar results were also observed by Timoteo et al. ${ }^{15}$.

A report from the GRACE registry showed that short-term and six-month mortality was increased significantly with higher admission glucose levels in patients across the whole spectrum of acute coronary syndromes. ${ }^{23}$ This association is probably mainly driven by an increased risk of early death, consistent with the paradigm that admission glucose level is a marker of stress rather than a reflection of a general glucometabolic state. ${ }^{24}$

Receiver-operator characteristic (ROC) were constructed using GRACE score and GRACE score + admission blood sugar of the patients with in-hospital death, which showed the sensitivity and specificity of GRACE score for predicting in-hospital death were found to be $79.4 \%$ and $58.1 \%$, respectively. Whereas after adding admission blood sugar value to GRACE score both the sensitivity and specificity increased to $82.4 \%$ and $58.6 \%$ respectively in this new model.

Logistic regression analysis of in-hospital mortality with independent risk factors showed GRACE score (e"155) + admission blood sugar (e"11.0 mmol/l) was more significantly associated with in-hospital mortality $(P=0.001$, $\mathrm{OR}=6.675,95 \% \mathrm{Cl} 2.366-13.610$ ). So, the new model better identifies those who do not have events than those who do. Thus the new model (with the addition of admission blood glucose to GRACE score) is better at identifying 'truly lowrisk' patients and is as good as in identifying patients who develop events. This might not be ideal when we are evaluating a risk score to identify high-risk patients. However, recent cardiovascular disease guidelines are encouraging a practice shift toward greater focus on identification of 'truly low-risk' patients instead of focusing on identification of highrisk patients. This allows a better selection of patients avoiding unnecessary interventions that might increase costs as well as the risk of procedure-related adverse events.

\section{Conclusion:}

Admission hyperglycemia is associated with increased mortality of patients with acute coronary syndrome. Therefore inclusion of admission blood glucose can add prognostic information to the established risk factors with the GRACE risk score.

\section{Reference:}

1. Islam AK, Majumder AA. Coronary artery disease in Bangladesh: a review. Indian Heart J 2013; 65(4): 424-35. 
2. Mehta RH, Califf RM, Garg J. The impact of anthropomorphic indices on clinical outcomes in patients with acute ST-segment Elevation Myocardial Infarction. Euro Heart J 2007; 28: 415-20.

3. Kannel WB, McGee DL. Diabetes and Cardiovascular Disease. JAMA 1979; 241: 2035-8.

4. Wahab NN, Cowden EA, Pearce NJ, Gardner MJ, Merry H, Cox JL. Is Blood Glucose an Independent Predictor of Mortality in Acute Myocardial Infarction in the Thrombolytic Era?. J Am Coll Cardiol 2002; 40: $1748-54$.

5. Ishihara M, Inoue I, Kawagoe T, Shimatani Y, Kurisu S \& Hata T et al. Is admission hyperglycaemia in nondiabetic patients with acute myocardial infarction a surrogate for previously undiagnosed abnormal glucose tolerance?. Eur Heart J 2006; 27: 2413-9.

6. Capes SE, Hunt D, Malmberg K, Gerstein HC. Stress hyperglycaemia and increased risk of death after myocardial infarction in patients with and without diabetes: a systematic overview. Lancet 2000; 356: 773-8.

7. Stranders I, Diamant M, van Gelder R, Spruijt H, Twisk JWR, Heine RJ, Visser FC. Admission blood glucose level as risk indicator of death after myocardial infarction in patients with and without diabetes mellitus. Arch Intern Med 2004; 164: 982-8.

8. Zeller M, Steg P, Ravisy J, Laurent Y, Janin-Manificat L, L'Huillier I, Beer J, Oudot A, Rioufol G, Makki H, Farnier M, Rochette L, Cottin Y. Prevalence and impact of metabolic syndrome on hospital outcomes in acute myocardial infarction. Arch Intern Med 2005; 165: 1192-98.

9. Oswald GA, Smith CCT, Betteridge DJ. Determinants and importance of stress hyperglycemia in nondiabetic patients with myocardial infarction. BMJ 1986; 293: 917-22.

10. Karlsberg RP, Cryer PE, Roberts R. Serial plama catecholamine response in the early course of acute myocardial infarction: relationship to infarct extent and mortality. Am Heart J 1981; 102: 24-9.

11. Meier JJ, Deifuss S, Klaman A. Plasma glucose at hospital admission and previous metabolic control determine myocardial infarct size and survival in patients with and without type 2 diabetes. Diabetes Care 2005; 28: 2551-3.

12. Takahashi T, Hiasa Y, Ohara Y. Acute hyperglycaemia prevents the protective effect or preinfarction angina on microvascular function after primary angioplasty for acute myocardial infarction. Heart Journal 2008; 94: $1402-6$.
13. Sala J, Masia R, Gonzalez de Molina FJ, FernandezReal JM, Gil M, Bosch D et al. REGICOR Investigators. Short-term mortality of myocardial infarction patients with diabetes or hyperglycaemia during admission. J Epidemiol Community Health, 2002: 56(9): 707-12.

14. Mudespacher D, Radovanovic D, Camenzind E, Essig M, Bertel O, Erne P, Eberli FR. Admission glycaemia and outcome in patients with acute coronary syndrome. Diabetes Vasc Dis Res, 2007: 4; 346-52.

15. Timoteo AT, Papoila AL, Rio P, Miranda F, Ferreira ML, Ferreira RC. Prognostic impact of admission blood glucose for all-cause mortality in patients with acute coronary syndromes: added value on top of GRACE risk score. Euro Heart J Acute Cardiovas Care, 2014: 3(3); 257-63.

16. Allison SP, Tomlin PJ, Chamberlain MJ. Some effects of anaesthesia and surgery on carbohydrate and fat metabolism. Br J Anaesth 1969; 41: 588-92.

17. Clarke RSJ, Johnston H, Sheridan B. The influence of anaesthesis and surgery on plasma cortisol, insulin and free fatty acids. Br J Anaesth 1970; 42: 295-9.

18. Oliver MF, Opie LH. Effects of glucose and fatty acids on myocardial ischaemia and arrythmias. Lancet 1994; 343: 155-8.

19. Holubarsch C, Ruf T, Goldstein DJ, et al. Existence of the Frank- Starling mechanism in the failing human heart: investigations on the organ, tissue, and sarcomere levels. Circulation 1996; 94: 683-9.

20. Marcus JT, Gotte MJ, Van Rossum AC, eta I. Myocardial function in infarcted and remote regions early after infarction in man: assessment by magnetic resonance tagging and strain analysis. Magn Reson Med 1997; 38: 803-10.

21. Tansey MJB, Opie LH. Plasma glucose on admission to hospital as a metabolic index of the severity of acute myocardial infarction. Can J Cardiol 1986; 2: 326-31.

22. Coutinho M, Gerstein HC, Wang Y, Yusuf S. The relationship between glucose and incident cardiovascular events: a metaregression analysis of published data from 20 studies of 95,783 individuals followed for $12 \cdot 4$ years. Diabetes Care 1999; 22: 233-40

23. Sinnaeve PR, Steg PG, Fox KAA, et al. Association of elevated fasting glucose with increased short-term and 6-month mortality in ST-segment elevation and nonST-segment elevation acute coronary syndromes. Arch Intern Med 2009; 169: 402-9.

24. Zeller M, Cottin $\mathrm{Y}$, Brindisi MC, et al. Impaired fasting glucose and cardiogenic shock in patients with acute myocardial infarction. Eur Heart J 2004; 25: 308-12. 